\title{
Three Attacks of Eclampsia occurring in a Multipara.
}

\author{
By A. J. Sturmer, Lieut.-Col. I.M.S., \\ Superintendent, Government Maternity Hospital, Madras.
}

Mrs. W., an Eurasian, commenced to menstruate in her 15th year, and was always regular, the flow lasting four or five days attended with pain in the lower abdomen on the first day. Discharge sometimes profuse. Micturition normal; said to be scanty during the pregnant state. Was married in her 19th year, and was admitted to the hospital in her 20th year pregnant with her first child in May, 1893. The membranes were ruptured artificially, and on the $23 \mathrm{rd}$ she was delivered of a live female child. She progressed favourably, and left the hospital on the 3rd of June.

She was re-admitted in August, 1895, pregnant for the second time, aged 22 years, in a semi-conscious state and in the ninth month of pregnancy. No uterine souffle, legs swollen; 16 ounces of urine were drawn by catheter and found to be loaded with albumen. She was apparently quite well when she went to bed, but had a fit during the evening and another this morning; before she could be removed from the carriage she had a third fit. This fit was followed by two athers, which were mitigated by the use of chloroform. The cervix was dilated by means of Champetier de Ribes' bag, and as the patient was very weak, the head was perforated and crushed with the cephalotribe. The foetal heart was doubtful on admission. The records are very deficient and only go as far as 5-45 p.m. on the day of delivery. During this time she had three more fits, and ether $3 \mathrm{ss}$ was injected fourteen times owing to her weak condition. She recovered and left the hospital on the 14th of September. The third and fourth confinements were natural in the hospital.

She was admitted on the 14 th of January, 1901, at 2-30 a.m., with a history of having had one fit at home and another on the way to the hospital. Labour commenced at midnight. She was about eight months pregnant; foetal heart doubtful; feet and hands very cedematous; urine loaded with albumen. At 4 a.m. she had an injection of morphia gr. $\frac{3}{4}$, having had two fits. At 9 a.m. a female child was stillborn, weighing 3lbs. 10oz. She had eight fits before delivery and six after. At 10-45 a.m. she had an injection of morphia gr. $\frac{1}{2}$. On the 15 th she had two more fits; albumen $1 / 12$ th; was very restless during the night; frequent twitchings of the hands 
and face; temperature $102^{\circ} \mathrm{F}$. Morphia gr. $\frac{1}{4}$ was repeated, and she was ordered a mixture of acetate and citrate of potash. On the 16th the urine was free from albumen; she passed a good night. The note made on the 17th is: "Restless; slept very little during the night, shouting and trying to get out of bed; complains of headache." On the 18th a trace of albumen was again noticed, but after this it quite disappeared; a mixture of chloral and bromide was given at bedtime. On the 22nd it is noted that she was quite sensible and able to answer questions. She continued to improve, and left the hospital on the 6th of February. The sixth confinement was also in the hospital, and was natural.

On the 12th of March, 1904, she was admitted for albuminuria, pregnant with her seventh child. The urine was found to be very scanty and loaded with albumen; both feet were œdematous. She was placed on a milk diet and given thyroid extract gr. 5 three times a day, and under this treatment the albumen quite disappeared and also the headache from which she at first suffered. She was advised to remain under observation until such time as labour set in, but she refused to stay in hospital, and left on the 21st of March. She was re-admitted on the 2nd of April of this year at 4-30 a.m., with a history of having had one fit at home, another on the way to the hospital and the third at the gate. Fetal heart audible; uterus corresponds to seven months; her upper garments were saturated with blood, due to her having bitten her tongue severely. Urine very scanty and loaded with albumen. On admission morphia gr. $\frac{1}{2}$ was injected, and shortly after thyroid extract gr. 10 was also administered, gr. 35 were subsequently given in divided doses. She had therefore in twelve hours 45 grains of thyroid. Saline injection under the breast one pint and acetate and citrate of potash mixture were also given. She had in all thirteen fits before delivery and none after. The cervix was dilated with a Barnes' bag, which was expelled as soon as it was put in. A male child was stillborn, weighing 3lbs. 12 ozs. On the day after delivery 46 ounces of urine were passed, containing about one-twelfth albumen. Her face was considerably more swollen on the second day, but after this the odema rapidly disappeared. She complained of headache up to the sixth day after delivery. The urine was examined on several occasions, but no casts were ever found. She left the hospital on the 21st of April, the nineteenth day after delivery, apparently quite well.

Remarks. In our experience of eclampsia two attacks occurring in the same patient are unknown. In the last ten years there have 
come under treatment 271 cases of eclampsia and in no single instance among these, except this case, has eclampsia occurred twice. Cases of recurrent eclampsia are not unknown in literature, but I imagine three attacks in the same individual are without record. The present case is a good example of the fact that the affection is not due to disease of the kidney. The microscopical and chemical examination of the urine show that when she left the hospital after her third attack there was no disease of the kidney, and if previous disease had been present it would in all probability have been accentuated. The swelling of the face after the disappearance of the fits has been noticed here in other cases, but why the face should swell, whilst the ædema of the extremities disappears I cannot say. In this case the swelling of the face was very much more marked on the second day, and the cedema of the lower extremities was fast disappearing. 


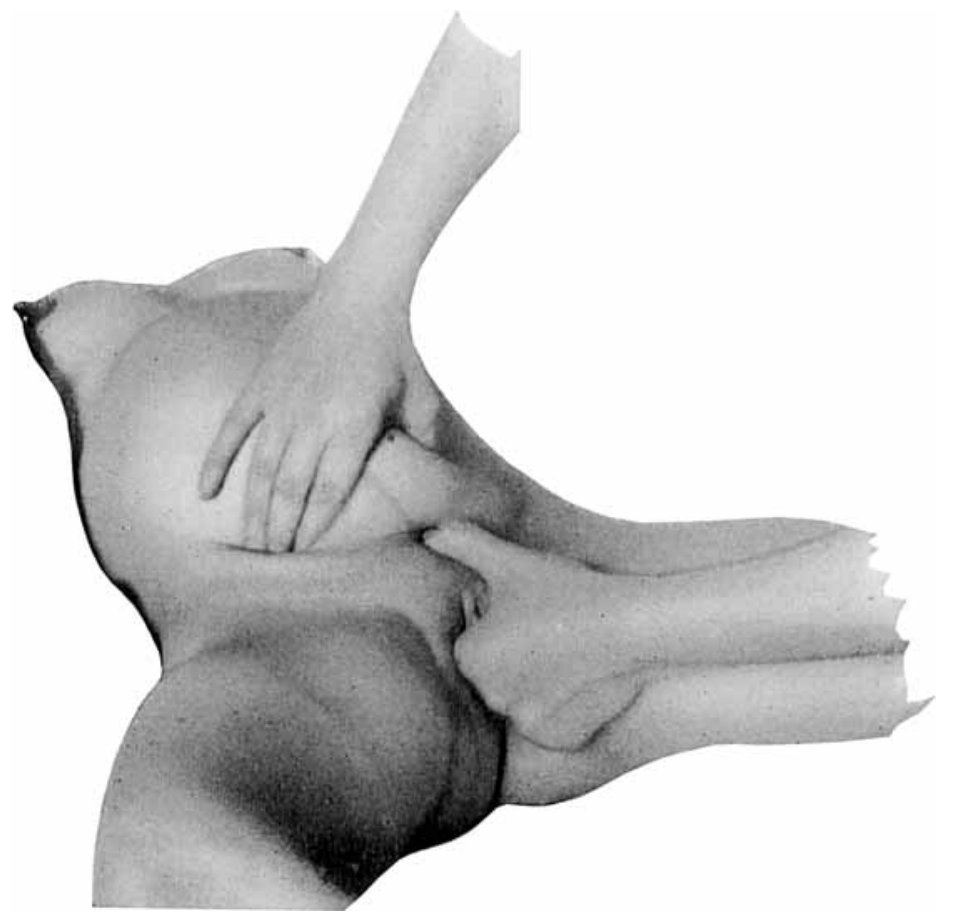

Author's Method.--Two fingers of the left hand are passed into the ragina and feel how much and in which manner the head engages, while the thumb of the same hand is moved along the brim and appreciates the amount of over-lapping. The right hand grasps the head by a Paulic grip and pushes it into the pelvis. 
